\title{
3D Object Volume Measurement Using Freehand Ultrasound
}

\author{
A.L. Bogush and A.V. Tuzikov \\ United Institute of Informatics Problems, \\ National Academy of Sciences of Belarus, \\ Surganova 6, 220012 Minsk, Belarus \\ \{bogush, tuzikov\}@mpen. bas-net.by \\ http://uiip.bas-net.by
}

\begin{abstract}
Algorithms for volume evaluation of 3D objects on freehand ultrasound images are considered. The position sensor used provides image spatial position and orientation data. The algorithms are based on Watanabe formula for volume computation and use cubic spline interpolation. They allow object volume evaluation on initial image sequence without reconstruction of 3D cube avoiding inevitable data loss at this pre-processing stage. The algorithm accuracy was tested on simulated and real objects.
\end{abstract}

\section{Introduction}

There are known various methods of 3D ultrasound image acquisition. The majority of them compose 3D images as a result of reconstruction from sequential 2D images. Recently special 3D probes have been developed, which are able to produce 3D images of a volume within a body. However, many technical problems need to be solved before such probes will be useful for routine clinical use.

Several different 3D ultrasound imaging approaches have been developed: mechanical scanners, free-hand techniques and 2D arrays [2]. In the last case 2D phased array of transducer elements is used to transmit a broad beam of ultrasound diverging away from the array, sweeping out a volume shaped like a truncated pyramid. However, it is still very expensive to use $2 \mathrm{D}$ arrays.

Three basic types of mechanical scanners have been developed: linear, tilt and rotational. They differ only in the manner ultrasound probe is moving, it can be translation, tilting or rotation. But for every type the scanning protocol is predefined. First 3D ultrasound systems based on mechanical movement of the probe were not very suitable for routine clinical applications because of restricted movement and angulations of the transducer.

Fortunately, new 3D ultrasound systems with free-hand acquisition using position sensor systems became available. Free-hand scanning techniques do not require a motorized fixture. In these approaches, a sensor is attached to the transducer to measure its position and orientation. Thus, the transducer can be held by the operator and be manipulated in the usual manner over the anatomy to be imaged. While the transducer is being manipulated, the acquired 2D images (see Fig. 1) are stored by a 
computer together with their spatial position and orientation. Several free-hand scanning approaches have been developed, making use of different sensing approaches: articulated arms, acoustic sensing, magnetic field sensing, optical sensing and image-based sensing. Most of recently documented systems use either an electromagnetic or an optical position sensor. In our application an electromagnetic position sensor, mounted on ultrasound probe was used (as it is shown in Fig. 2).

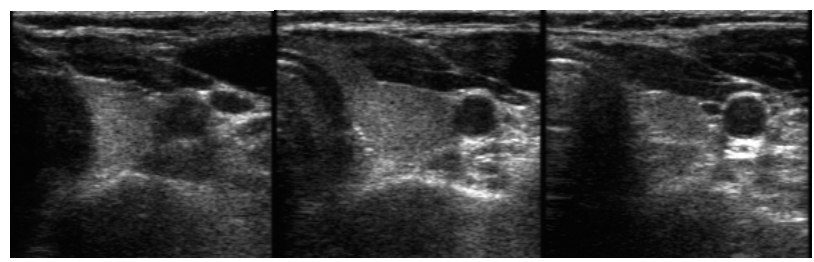

Fig. 1. An example of sequence of thyroid gland ultrasound images

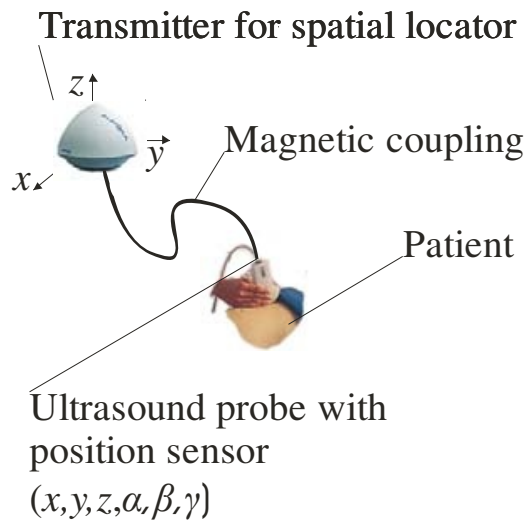

Fig. 2. Obtaining position and orientation of ultrasound images

The majority of freehand 3D ultrasound systems interpolate these data into a 3D regular array before doing any operations.

Following the approach proposed in [3] we proposed a volume measurement algorithm from non-parallel cross-sections. This report is devoted to volume estimation algorithm based on Watanabe formula [1] and uses an interpolation by cubic splines. The same splines are applied also for computation of object area and centroid in every cross-section. One can outline object under interest by cubic spline in several non-parallel cross-sections (Fig. 3). We use non-parallel ultrasound images directly without reconstruction to the regular 3D array, and therefore we can overcome data loss at interpolation stage. We implemented several options for object segmentation. The basic option is direct using of non-parallel planes representing initial ultrasound images, as shown in Fig. 4a). Another method is using planes passing through a common pre-defined axis (Fig 4b). This axis is chosen manually. In this case plane images are interpolated from initial cross-section images. This case is 
better for visual detecting of object boundaries than the first one. One outlines the object boundaries in these planes and then gets object volume estimation. The third option is derived from the second one. Object outlines for the case with the chosen axis generate outlines in the parallel sections orthogonal to the axis (see Fig 4c).

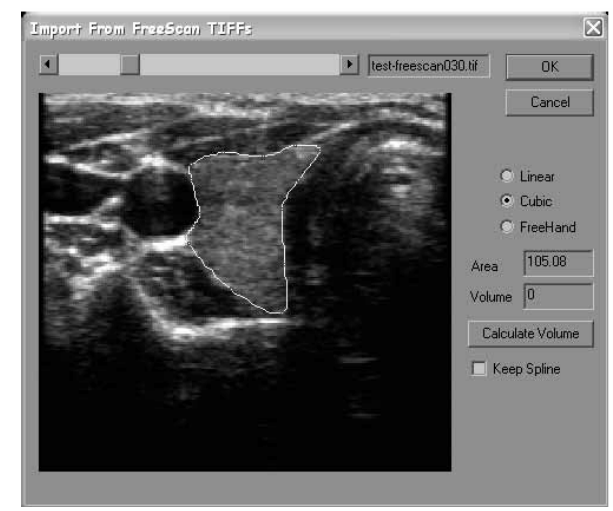

Fig. 3. Thyroid gland outlining in cross-sections

The method proposed is developed for improvement of earlier diagnostics of various diseases, and thyroid gland cancer in particular. This goal can be achieved by decreasing the error level of volume computation from ultrasound data.

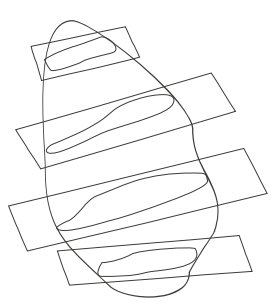

a)

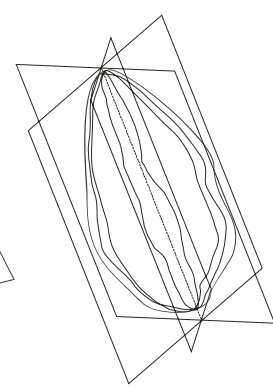

b)

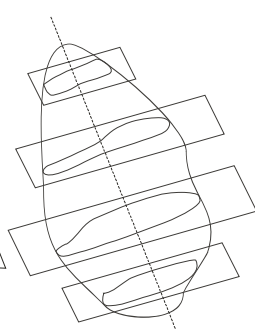

c)

Fig. 4. Various methods of object outlining: a) non-parallel cross-sections, b) planes with common axis, c) parallel cross-sections

\section{Method Description}

A new method for volume computation from non-parallel cross-sections was suggested and investigated in [3,4]. This method uses a cubic spline interpolation [5]. The method was discussed in details for 2D representation (so called 2D cubic planimetry) and it was mentioned how it can be implemented for the 3D 
representation. However, no concrete formulae were given there for the 3D representation. We proposed in [6] the explicit formulae for volume estimation. Suppose that object boundary outlines are obtained in sections $1,2, \ldots, N$. Denote by $V_{i}$ the object volume between cross-sections $i$ and $i+1$. Assuming that cross-section area is changing according to cubic interpolating spline, for every object part between two cross-sections one has the following formula for volume computation [6]:

$$
V_{i}=\mathbf{x}_{i} A \mathbf{u}_{i}^{t}+\mathbf{y}_{i} A \mathbf{v}_{i}^{t}+\mathbf{z}_{i} A \mathbf{w}_{i}^{t}
$$

where

$$
A=\frac{1}{240}\left(\begin{array}{cccc}
0 & -11 & 12 & -1 \\
11 & -120 & -143 & 12 \\
-12 & 143 & 120 & -11 \\
1 & -12 & 11 & 0
\end{array}\right)
$$

Here we use the notation $\mathbf{x}_{i}=\left(x_{i-1}, x_{i}, x_{i+1}, x_{i+2}\right)$. Similarly are defined $\mathbf{y}_{i}, \mathbf{z}_{i}, \mathbf{u}_{i}, \mathbf{v}_{i}, \mathbf{w}_{i}$. Note that $\vec{c}_{i}=\left(x_{i}, y_{i}, z_{i}\right)$ denotes coordinates of the $i$-th object crosssection centroid and $\vec{s}_{i}=\left(u_{i}, v_{i}, w_{i}\right)$ are coordinates of vector, orthogonal to the $i$-th cross-section and having the length equal to the object area in the cross-section. It is also assumed that index 0 is replaced by 2 and $N+1$ is replaced by $N-1$.

The volume of the whole object is computed as follows:

$$
V=\left|\sum_{i=1}^{N-1} V_{i}\right|
$$

In the case of parallel cross-sections (see Fig 4c)) the volume does not depend on cross-section centroid coordinates and equals to the integral of the cross-section area:

$$
V=\left|\int s(h) d h\right| .
$$

Here $s(h)$ is a function of cross-section areas parameterized by a position $h$ of an intersection point of planes with some orthogonal line.

Denote as before by $\mathbf{h}_{i}=\left(h_{i-1}, h_{i}, h_{i+1}, h_{i+2}\right)$ and $\mathbf{s}_{i}=\left(s_{i-1}, s_{i}, s_{i+1}, s_{i+2}\right)$ the values of the parameter and the corresponding cross-section area values used for generating the $i$-th spline segment. In this case scalar values of areas are interpolated and the following formula is valid for volume $V_{i}$ :

$$
V_{i}=\mathbf{h}_{i} A \mathbf{s}_{i}^{t},
$$

with the same matrix $A$ as in (2).

Another approach called multiplanar volume approximation is implemented in FreeScan software [7]. Using this method, the longest diameter of a 3D object is determined manually and this diameter is used as a rotation axis. Then nine planes, 
rotated around this axis with a fixed angle are automatically generated. The border of the object in each plane is outlined manually and the final volume is evaluated based on these outlines. The evaluation approach used creates equidistant planes orthogonal to the rotation axis. In every equidistant plane a cubic spline is created automatically based on intersection points of outlines with this plane. Then the areas of regions bounded by cubic splines are calculated. The volume results from the areas multiplied by the distance between planes.

We extended this idea in two ways. Firstly, our method using formula (1) can be applied directly to planes, rotated around axis as shown in Fig 4b). One can choose number of planes and their rotation angles around the axis, in order to see better the organ under investigation.

Secondly, parallel cross-sections can be reconstructed automatically from outlines done in planes, rotated around the common axis. The points defining the object boundary are generated for every cross-section. These points are used as spline control points.

We proposed in [8] the explicit formulae for computation of area and low order geometric moments for object bounded by a uniform spline curves. For interpolating spline curve the following formulae hold.

Let us denote

$$
\begin{aligned}
& p_{i 1}=x_{i} y_{i+1}-x_{i+1} y_{i}, p_{i 2}=x_{i} y_{i+2}-x_{i+2} y_{i}, \\
& p_{i 3}=x_{i} y_{i+3}-x_{i+3} y_{i}, p_{i 4}=x_{i+1} y_{i+3}-x_{i+3} y_{i+1} .
\end{aligned}
$$

where $\left(x_{i}, y_{i}\right)$ are a control point coordinates in the local plane coordinate system.

Then the area $S$ (up to sign) and first order moments $m_{01}$ and $m_{10}$ are computed as follows:

$$
\begin{aligned}
& S=\frac{1}{240} \sum_{i=1}^{n}\left(-165 p_{i 1}+24 p_{i 2}-p_{i 3}\right) \\
& m_{10}=\frac{1}{6720} \sum_{i=1}^{n}\left(-p_{i 1} 1643\left(x_{i}+x_{i+1}\right)\right. \\
& +p_{i 2}\left(302 x_{i+1}+15 x_{i+3}+136\left(x_{i}+x_{i+2}\right)\right) \\
& \left.+p_{i 3}\left(8\left(x_{i}+x_{i+2}\right)-x_{i}-x_{i+3}\right)-p_{i 4} 15 x_{i}\right) \\
& m_{01}=\frac{1}{6720} \sum_{i=1}^{n}\left(-p_{i 1} 1643\left(y_{i}+y_{i+1}\right)\right. \\
& +p_{i 2}\left(302 y_{i+1}+15 y_{i+3}+136\left(y_{i}+y_{i+2}\right)\right) \\
& \left.+p_{i 3}\left(8\left(y_{i}+y_{i+2}\right)-y_{i}-y_{i+3}\right)-p_{i 4} 15 y_{i}\right)
\end{aligned}
$$

Centroid coordinates are computed using the first order moments $m_{01}$ and $m_{10}$ :

$$
\left(x_{c}, y_{c}\right)=\left(\frac{m_{10}}{S}, \frac{m_{01}}{S}\right)
$$




\section{Volume Computation Algorithm}

Below we summarize the algorithm for volume computation from non-parallel crosssections.

\section{Algorithm 1}

Input: A sequence of planes $\Pi_{i}$ with normal vectors $\vec{n}_{i}, i=1,2, \ldots, N$.Position of every plane is fixed in a global coordinate system. In a local coordinate system of every plane $\Pi_{i}$ there are given control points $\mathbf{p}_{1}, \mathbf{p}_{2}, \ldots, \mathbf{p}_{n_{i}}{ }^{\circ}$ which define a spline representing the object boundary. These control points are obtained as a result of a segmentation procedure performed, for example, manually.

Output: Volume $V$ of the object.

1. For every plane $\Pi_{i}$ in a local coordinate system compute object area $S_{i}$ and centroid coordinates $\left(x_{c_{i}}, y_{c_{i}}\right)$ using formulae (5) and (8).

2. Compute global spatial coordinates of centroids $\vec{c}_{i}, i=1,2, \ldots, N$, using the plane coordinates and orientation in the global coordinate system.

3. Given a set of vectors $\vec{s}_{i}=S_{i} \vec{n}_{i}$ and centroids $\vec{c}_{i}, i=1,2, \ldots, N$ compute the volume using formulae (1) and (3).

It is clear that Algorithm 1 can be applied directly for the case when 2D images have a common axis. One has to take into account that outlined cross-sections are divided into two parts separated by the axis and sorted according to changing of the rotational angle.

\section{Experiments Results}

To test the accuracy of Algorithm 1 using formulae (1) and (3) we used simulated 3D objects, physical rubber and silicon phantoms.

The tested simulated 3D images (see Fig. 5) have size $200 \times 200 \times 200$. The exact volumes of these objects were computed in advance. Non-parallel cross-sections were generated randomly for any given number of sections. We have chosen randomly the.

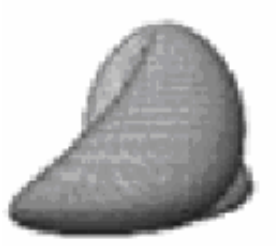

a)

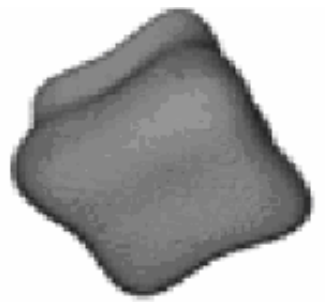

b)

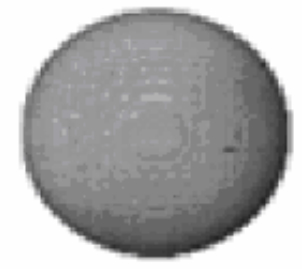

c)

Fig. 5. Simulated 3D objects used for volume evaluation 


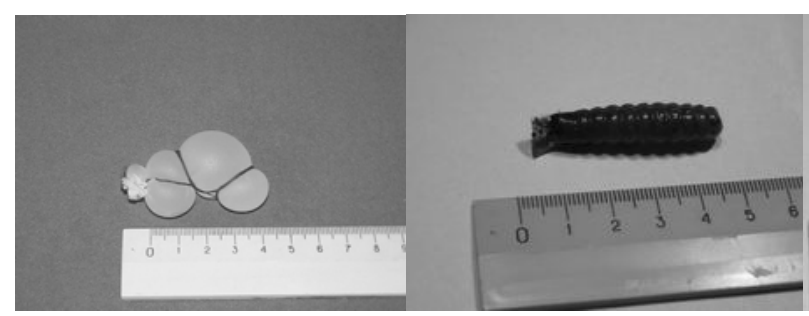

a) b)

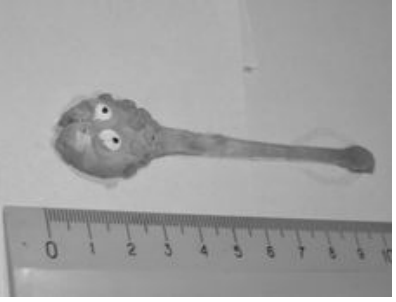

c)

Fig. 6. Examples of physical phantoms used for volume evaluation

distance between cross-sections and rotation angle around initial positions (up to $15^{\circ}$ ) The object volume was computed using Algorithm 1 for every realization. Then volume mean and standard deviation errors were calculated for these realizations.

At the next step, we used rubber balls and silicon phantoms, as shown in Fig. 6. They were scanned several times with ultrasound scanner and position sensor mounted on it. The original object volume was measured using measuring glasses with accuracy of 0.25-2.5 $\mathrm{ml}$ depending on object sizes.

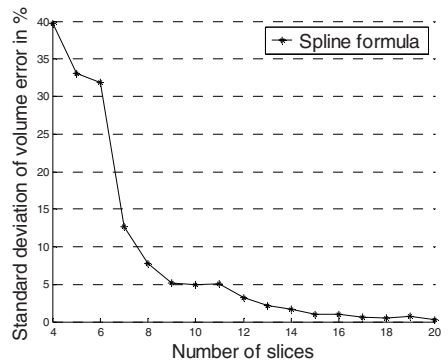

a)

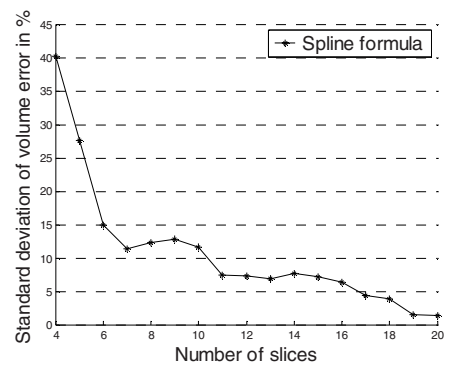

c)

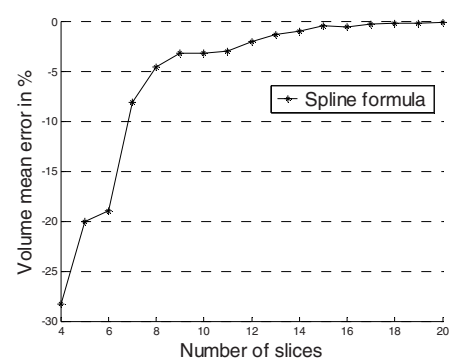

b)|

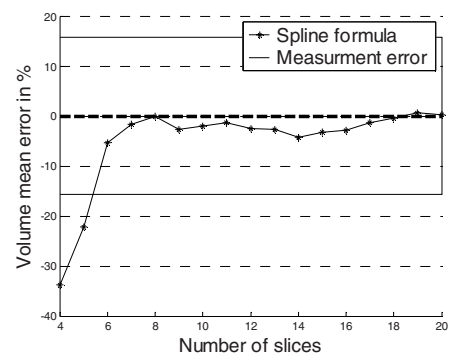

d)

Fig. 7. Standard deviation and volume mean errors vs number of cross-sections computed for simulated object shown in Fig. 5a) (a),b)) and fantom shown in Fig 6a) (c),d))

The plots of the data obtained for simulated 3D object shown in Fig 5a) are presented in Fig. 7-9a) and b). The example of resulting plots for object shown in 


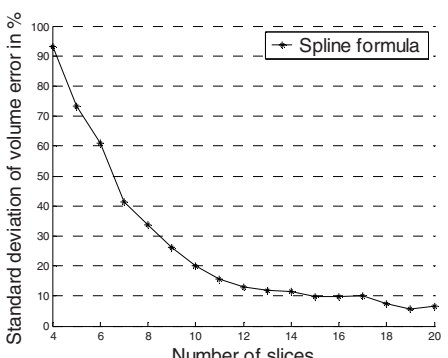

a)

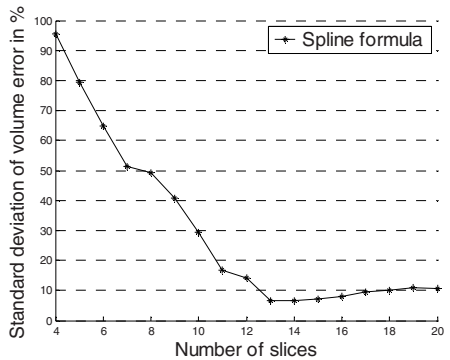

c)

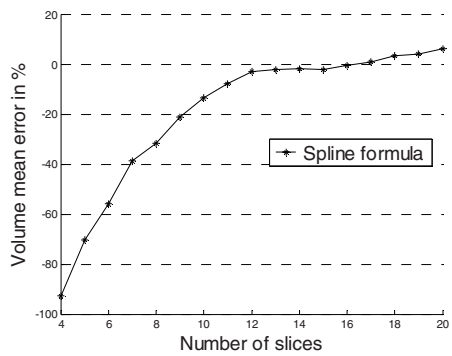

b)

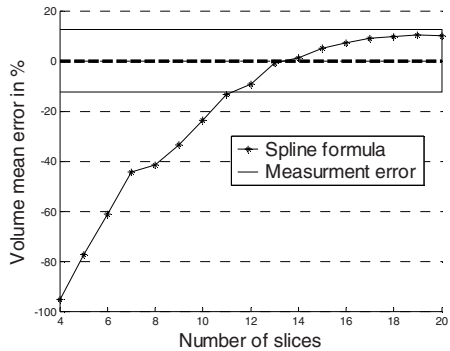

d)

Fig. 8. Standard deviation and volume mean errors for objects shown in Fig. 5a) (a), b)) and Fig 6a) (c), d)) for cross-sections with common axis

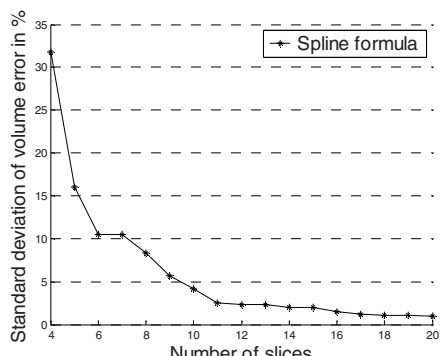

a)

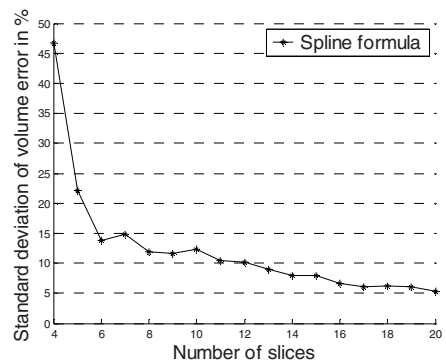

c)

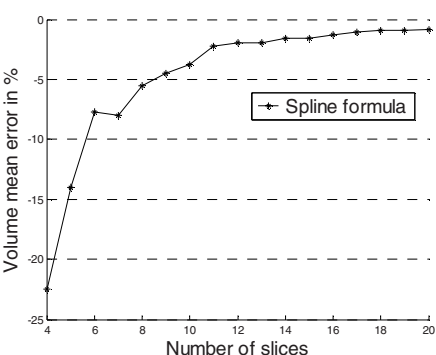

b)

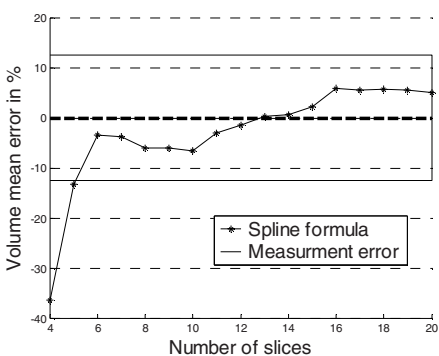

d)

Fig. 9. Standard deviation and volume mean errors for objects shown in Fig. 5a) (a), b)) and Fig 6a) (c), d)) for parallel cross-sections 
Fig 6a) are shown in Fig 7-9c) and d). The measurement accuracy limits are shown by solid lines.

When using the current approach volume is under-estimated for low cross-section count. As one may see in Fig. 7, error level of 5\% is reached at 8-10 outlined crosssections. It follows from Fig. 8, that volume measurement results with a common cross-sections are more sensitive to the number of cross-sections. This property should be explained and verified more carefully. One has to take also into account both measurement data acquisition errors. However, using this method one can detect more valuable tissue properties and outline object boundaries more accurately (compare for example a radial cross-section in Fig. 10 and the corresponding initial images of the same object in Fig. 1). Results for parallel cross-sections (Fig. 9) are similar to original, but slightly noisier due to conversion error.

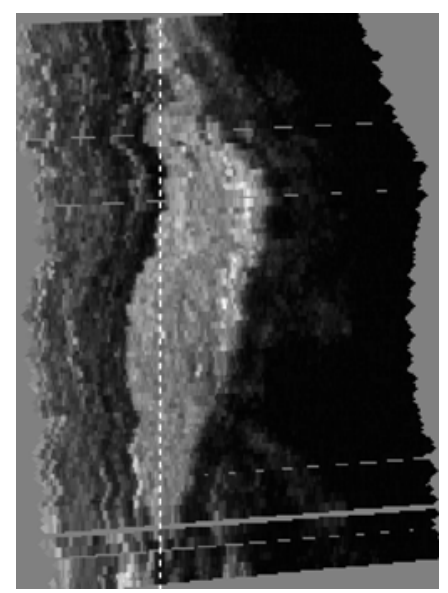

Fig. 10. Radial cross-section

\section{Conclusion}

We proposed several algorithms for the 3D object volume estimation from a series of non-parallel 3D freehand ultrasound images.

The algorithms were tested for a number of simulated and real objects with known volumes. The dependence of volume errors on the number of cross-sections was studied. For these quite simple objects it was usually sufficient for 8 cross-sections to obtain a mean volume error less than $5 \%$.

We plan to use our algorithm as a tool in early diagnostics of thyroid cancer with 3D ultrasound because it can allow more accurate determination of progressive growth of cancer nodules as compared with benign ones.

The work was done in framework of the ISTC B-517 project. 


\section{References}

1. Y. Watanabe. A method for volume estimation by using vector areas and centroids of serial cross sections. IEEE Trans. Biomed. Eng., 29:202-205, 1982.

2. A. Fenster, D. Downey, and H. Cardinal. Three-dimensional ultrasound imaging. Physics in Medicine and Biology, 46:67-99, 2001.

3. G.M. Treece, R.W. Prager, A.H. Gee, and L. Berman. Fast surface and volume estimation from non-parallel cross-sections for freehand 3-D ultrasound. Medical Image Analysis, 3(2):141-173, 1999.

4. G. Treece. Volume measurement and surface visualisation in sequential freehand $3 D$ ultrasound. PhD thesis, University of Cambridge, Department of Engineering, 2000.

5. E. Catmull and R. Rom. A class of local interpolating splines. In R. Barnhill and R. Risenfeld, editors, Computer Aided Geometric Design, pages 317-326. Academic Press, San Francisco, 1974.

6. S.A. Sheynin, A.V. Tuzikov, A.L. Bogush. Improvements of Volume Computation from Non-parallel Cross-sections. 17th International Conference on Pattern Recognition ICPR'2004, 23-26 August 2004, Cambridge, UK, vol. 4, 815-818.

7. S. Schlogl, E. Werner, M. Lassmann, J. Terekhova, S. Muffert, S.Seybold, and C. Reiners. The use of three-dimensional ultrasound for thyroid volumetry. Thyroid, 11(6):569-574, 2001.

8. S. Sheynin and A. Tuzikov. Moment computation for objects with spline curve boundary. IEEE Transactions on Pattern Analysis and Machine Intelligence, vol. 25 no. 10:13171322, 2003. 